\title{
Effect of fluence smoothening in intensity modulated radiotherapy planning and delivery
}

\author{
B. Rekha Reddy ${ }^{1}$, C. Varatharaj ${ }^{2}$, M. Ravikumar ${ }^{3}$, K. M. Ganesh ${ }^{4}$ \\ ${ }^{1}$ Smt. B. Rekha Reddy, Research Scholar, ${ }^{2}$ Dr. C. Varatharaj, Associate Professor, ${ }^{3}$ Dr. M. Ravikumar, Professor, \\ ${ }^{4}$ Dr. K.M. Ganesh, Professor, all authors are affiliated with Department of Radiation Physics, Kidwai Memorial Institute \\ of Oncology, Hosur Road, Bangalore, Karnataka, India.
}

Corresponding Author: Dr. C. Varatharaj, Associate Professor, Department of Radiation Physics, Kidwai Memorial Institute of Oncology, Hosur Road, Bangalore, Karnataka, India. Email: drvaratharaj@gmail.com

\begin{abstract}
Objective: In Intensity Modulated Radiotherapy (IMRT) planning, optimization is a computational problem, potentially susceptible to noise and artifacts (high frequency spatial fluctuations) producing sharp fluence peaks and valleys in millimetric spatial scale. A solution to this problem is to smooth the beam profiles. Methods and Materials: In the Eclipse TPS, fluence smoothing is achieved within the objective function of the inverse treatment planning systems. Plans were developed for a $6 \mathrm{MV}$ photon beam from a Varian Clinac-DHX equipped with a 120 leaves MLC. Total of 160 dose plans were compared and fed into the analysis process. The dose plan quality has been analysed in terms of Statistical computation by means of two-sided paired t-test between two smoothening levels (s25 and s75) in terms of Homogeneity-Index, Conformity-Index, Target dose coverage and OAR dose differences in terms of max, min and mean doses. Results: From our present study on the influence of smoothening of fluence levels in IMRT plans results, there was a reduction in total MU's with no significant statistical variation in terms of mean differences of HI Index, CI index, PTV coverages, OAR doses. Moreover, the reduction in MU's will help the less head leakage dose hence the lower whole-body dose, which will help the patient to reduce the chances for secondary malignancies. Conclusion: Hence, we conclude that, higher fluence smoothening levels with acceptable difference in target coverage and minimum variation of OAR should be selected for the execution.
\end{abstract}

Keywords: Radiotherapy, IMRT, Fluence, OAR

\section{Introduction}

Intensity-modulated radiation therapy (IMRT) is rapidly gaining acceptance as a practical and efficient method for generating dose distributions whose conformality and critical organ sparing far exceed that of traditional 3D-CRT [1]. It is argued that the increased conformality will lead to improved local control and higher long-term survival rates as well as better quality of life for the patient.

IMRT consists of two key components: an inverse planning or optimization algorithm to calculate the "optimal" beam profiles and a delivery system to generate them. In the process of optimization, algorithm is a mathematical objective function, which is an attempt to quantify the clinical objectives and assign a numerical score to each plan. Literature offers a huge

Manuscript received: $7^{\text {th }}$ July 2018

Reviewed: $17^{\text {th }}$ July 2018

Author Corrected: $24^{\text {th }}$ July 2018

Accepted for Publication: $27^{\text {th }}$ July 2018 variety of studies, at planning or clinical level, where a plethora of inverse planning algorithms have been investigated [2-5] to explore IMRT performances under several points of view. The optimisation process is a computational problem, potentially susceptible to noise and artifacts (high frequency spatial fluctuations) producing sharp fluence peaks and valleys in millimetric spatial scale. [6] A solution to this problem is to smooth the beam profiles.

There are two methods of doing so: a) smoothing can be applied outside the objective function, i.e., after the "optimal" profile has been produced by the optimization process, or b) the smoothness of the profiles can be included in the objective function as a criterion in the optimization process. The first method is easier to implement and allows the use of different smoothing algorithms and filters. However, it suffers from a fundamental drawback: as smoothing is applied 


\section{Original Research Article}

outside the objective function, its dosimetric effect is not considered in the optimization process. Therefore, the method cannot differentiate between those features of the beam profile (peaks, valleys, and gradients) that are clinically relevant and those that are not, i.e., those that are computational artefacts. In the second method, in contrast, the "unsmoothness" of the profile negatively affects the cost function, so that its dosimetric effect is incorporated in the optimization process [7]. In the present study, we investigated the interplay between fluence complexity, dose calculation algorithms, dose calculation spatial resolution and delivery characteristics (monitor units, and dose delivery against dose prediction agreement).

A sample set of complex planning cases were selected and tested using a commercial treatment planning system capable of inverse optimization and equipped with tools to tune fluence smoothness.

\section{Materials and Methods}

Planning Design: Plans were designed using the Eclipse TPS from Varian (release 8.6.11) and its inverse Dose Volume Optimizer (DVO). Plans were developed for a $6 \mathrm{MV}$ photon beam from a Varian Clinac-DHX equipped with a 120 leaves MLC at our Institute. In the Eclipse TPS, fluence smoothing is achieved within the objective function of the inverse treatment planning systems. Ten IMRT planning cases (seven head and neck and three cervix) were selected as representative of demanding planning requirements. All the beams were coplanar. Leaf sequencing and delivery are based on the dynamic sliding window technique.

Fluence Description: In Eclipse, optimal fluence smoothing is part of the DVO algorithm and it is performed along two directions: X, parallel to the MLC movement and $\mathrm{Y}$, orthogonal to it. Smoothing is applied at each optimisation iteration by adding two smoothness-related planning objectives in the cost function that account for the difference between neighbouring fluence values. To appraise the effectiveness of fluence smoothing and its interplay with other planning variables, the study was organised performing full optimisation and dose calculation for all the combinations of the following three variables:

1) Smoothing parameters: X- and Y-Smooth described above are the smoothening parameter ' $s$ ' in the following were varied simultaneously and set to 25,50 , 75 and 100 (s25, s50, s75 and s100 in the following) being the higher the values the higher the smoothing of the fluence patterns. Routinely, in clinical practice, $\mathrm{X}$ and $\mathrm{Y}$ - Smooth are set in the range 0-100.

2) Dose calculation algorithm: two algorithms were used: the Single Pencil Beam Algorithm (PBC), and the newly introduced convolution/superposition algorithm Anisotropic Analytical Algorithm (AAA).

3) Spatial resolution of dose calculation matrix: two grids were used: 2.5 (the minimum grid for PBC) and 5 $\mathrm{mm} .2 .5 \mathrm{~mm}$ is also the internal grid size used by Eclipse to compute and store fluences.

For each case (a combination of the three above variables, for a total of 16 plans per case) optimisation was carried out using a fixed set of dose volume objectives. Total of 160 dose plans were compared and fed into the analysis process.

Statistical Computation methods: The dose plan quality has been analysed in terms of mean differences (Statistical computation by means of two-sided paired ttest) between two smoothening levels (s25 and s75) in terms of Homogeneity index (HI index), Conformity index (CI Index), Target dose coverages (Maximum and Minimum dose differences) and OAR dose differences in terms of Max, min and Mean doses. (OAR1 was spinal cord for $\mathrm{H} \& \mathrm{~N}$ plans and bladder for cervix cases. OAR 2 was brain stem and rectum for $\mathrm{H} \& \mathrm{~N}$ and Cervix cases respectively). Delivery reliability was investigated by means of standardised pre-treatment verification methods using I'matri XX for s25 and s75 fluence levels with 0.25 spatial resolutions for both $\mathrm{PBC}$ and AAA algorithms, therefore a total of 40 pretreatment verification plans were calculated in Eclipse TPS and compared with measured fluence.

\section{Results}

In comparison of monitor units among different smoothening levels with respect to $\mathrm{s} 25$ level as $100 \%$, the monitor units reduces while increasing the fluence levels. The Figure 1. and 2 shows the monitor unit comparison between different fluence levels for the grid size of 0.25 and 0.5 respectively. In the combination of PBC algorithm with 0.25 grid size, the total mean MU decreases from $1143 \pm 211$ to $992 \pm 210$ for s25 and s100 respectively. Similarly, AAA with 0.25 has reduced from $1161 \pm 210$ to $999 \pm 241$ for s25 and s100 respectively. For the grid size of 0.5 , PBC algorithm it was varied from $1172 \pm 234$ to $979 \pm 220$ and for AAA algorithm the variation was $1202 \pm 230$ to $993 \pm 238$ for the smoothening levels of s 25 and s100 respectively. The comparison between the grid size of 0.25 and 0.5 for PBC and AAA algorithms were shown in Figure 3. 
Original Research Article



Figure-1: Comparison of PBC and AAA calculation algorithm for the grid size of 0.25 in terms of monitor units between different fluence smoothening levels

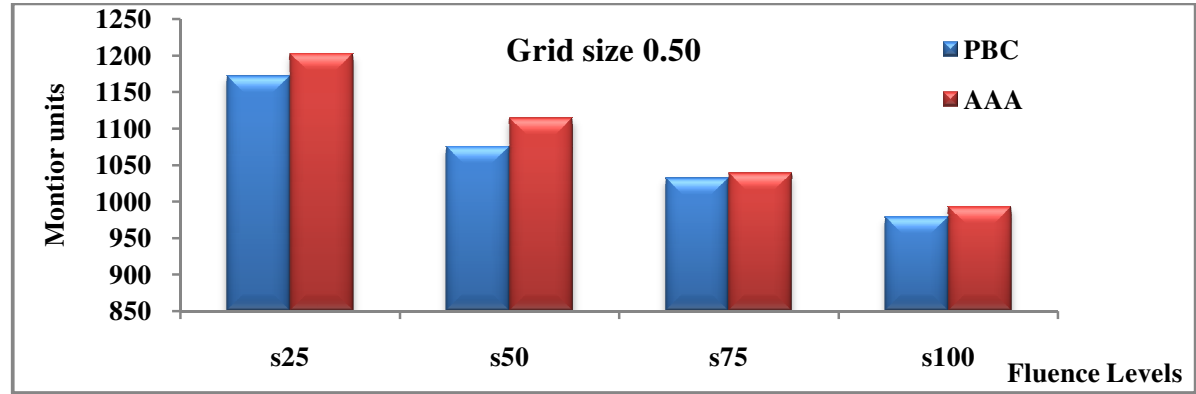

Figure- 2: Comparison of PBC and AAA calculation algorithm for the grid size of 0.5 in terms of monitor units between different fluence smoothening levels.



Figure- 3: Comparison between the calculation grid size of 0.25 and 0.5 for PBC and AAA algorithms in terms of monitor units.

Table-1: Difference of $\mathrm{HI}$ index between fluence smoothening levels of s25 and s75 for PBC and AAA algorithms for the grid size of 0.25 .

\begin{tabular}{|c|c|c|c|c|c|c|}
\hline \multirow[t]{2}{*}{ S. No. } & \multicolumn{3}{|c|}{ PBC } & \multicolumn{3}{|c|}{$\mathbf{A A A}$} \\
\hline & s25 (\%) & s75 (\%) & Difference & s25 (\%) & s75 (\%) & Difference \\
\hline 1 & 5.39 & 5.32 & 0.07 & 6.77 & 6.53 & 0.24 \\
\hline 2 & 5.71 & 6.52 & -0.81 & 7.60 & 8.04 & -0.44 \\
\hline 3 & 6.61 & 6.65 & -0.04 & 7.07 & 7.15 & -0.08 \\
\hline 4 & 6.01 & 6.63 & -0.62 & 6.46 & 7.29 & -0.83 \\
\hline 5 & 5.50 & 5.58 & -0.08 & 8.38 & 8.53 & -0.15 \\
\hline 6 & 6.01 & 5.97 & 0.04 & 6.53 & 6.37 & 0.16 \\
\hline 7 & 5.24 & 5.04 & 0.20 & 8.98 & 8.90 & 0.08 \\
\hline 8 & 7.08 & 7.17 & -0.09 & 7.30 & 7.23 & 0.07 \\
\hline 9 & 5.55 & 5.58 & -0.03 & 5.98 & 6.00 & -0.02 \\
\hline 10 & 2.84 & 2.74 & 0.10 & 2.60 & 2.60 & 0.00 \\
\hline \multicolumn{3}{|c|}{ MEAN } & -0.13 & & & -0.10 \\
\hline \multicolumn{3}{|c|}{ SD } & 0.33 & & & 0.32 \\
\hline \multicolumn{3}{|c|}{ p value } & 0.25 & & & 0.36 \\
\hline
\end{tabular}


Table-2: Difference of $\mathrm{HI}$ index between fluence smoothening levels of $\mathrm{s} 25$ and $\mathrm{s75}$ for PBC and AAA algorithms for the grid size of 0.5 .

\begin{tabular}{|c|c|c|c|c|c|c|}
\hline \multirow{2}{*}{ S. No. } & \multicolumn{5}{|c|}{ PBC } & \multicolumn{2}{c|}{ AAA } \\
\cline { 2 - 7 } & $\mathbf{s 2 5}(\%)$ & $\mathbf{s 7 5}(\%)$ & Difference & s25 (\%) & s75 (\%) & Difference \\
\hline 1 & 5.21 & 5.00 & 0.21 & 9.04 & 8.81 & 0.23 \\
\hline 2 & 5.87 & 6.98 & -1.11 & 9.80 & 10.78 & -0.98 \\
\hline 3 & 6.97 & 6.47 & 0.50 & 7.31 & 7.09 & 0.23 \\
\hline 4 & 6.01 & 7.17 & -1.16 & 7.98 & 9.18 & -1.20 \\
\hline 5 & 6.20 & 6.43 & -0.23 & 11.37 & 11.68 & -0.31 \\
\hline 6 & 5.80 & 5.76 & 0.04 & 7.01 & 7.01 & 0.00 \\
\hline 7 & 5.29 & 5.11 & 0.18 & 6.27 & 6.24 & 0.03 \\
\hline 8 & 7.43 & 7.39 & 0.04 & 9.28 & 9.29 & -0.01 \\
\hline 9 & 5.76 & 5.78 & -0.02 & 7.40 & 7.33 & 0.07 \\
\hline 10 & 2.94 & 3.00 & -0.06 & 3.10 & 3.04 & 0.06 \\
\hline
\end{tabular}

Table-3: Difference of $\mathrm{CI}$ index between fluence smoothening levels of $\mathrm{s} 25$ and $\mathrm{s75}$ for PBC and AAA algorithms for the grid size of $\mathbf{0 . 2 5}$.

\begin{tabular}{|c|c|c|c|c|c|c|}
\hline \multirow{2}{*}{ S. No. } & \multicolumn{5}{|c|}{ PBC } & \multicolumn{2}{c|}{ AAA } \\
\cline { 2 - 7 } & $\mathbf{s 2 5}(\%)$ & $\mathbf{s 7 5}(\%)$ & Difference & $\mathbf{s 2 5}(\%)$ & $\mathbf{s 7 5}(\%)$ & Difference \\
\hline 1 & 2.29 & 2.36 & -0.07 & 2.27 & 2.34 & -0.07 \\
\hline 2 & 1.75 & 1.72 & 0.03 & 1.38 & 1.38 & 0.03 \\
\hline 3 & 1.40 & 1.41 & -0.02 & 1.18 & 1.16 & 0.02 \\
\hline 4 & 1.20 & 1.17 & 0.03 & 1.24 & 1.27 & -0.03 \\
\hline 5 & 1.23 & 1.26 & -0.03 & 1.96 & 1.98 & -0.02 \\
\hline 6 & 1.99 & 2.00 & -0.01 & 1.44 & 1.46 & -0.02 \\
\hline 7 & 1.40 & 1.43 & -0.03 & 1.10 & 1.10 & 0.00 \\
\hline 8 & 1.11 & 1.11 & 0.00 & 1.08 & 1.08 & 0.00 \\
\hline 9 & 1.09 & 1.08 & 0.01 & 1.96 & 2.04 & -0.08 \\
\hline 10 & 1.96 & 2.04 & -0.08 & -0.02 & & -0.02 \\
\hline
\end{tabular}


Table-4: Difference of CI index between fluence smoothening levels of s25 and s75 for PBC and AAA algorithms for the grid size of 0.5 .

\begin{tabular}{|c|c|c|c|c|c|c|}
\hline \multirow{2}{*}{ S. No. } & \multicolumn{3}{|c|}{ PBC } & \multicolumn{3}{|c|}{$\mathbf{A A A}$} \\
\hline & s25 (\%) & s75 (\%) & Difference & s25 (\%) & s75 (\%) & Difference \\
\hline 1 & 2.23 & 2.35 & -0.12 & 2.21 & 2.27 & -0.06 \\
\hline 2 & 1.79 & 1.79 & 0.00 & 2.35 & 2.28 & 0.07 \\
\hline 3 & 1.39 & 1.39 & 0.00 & 1.40 & 1.39 & 0.00 \\
\hline 4 & 1.18 & 1.16 & 0.02 & 1.14 & 1.14 & 0.00 \\
\hline 5 & 1.22 & 1.23 & -0.01 & 1.21 & 1.25 & -0.04 \\
\hline 6 & 1.93 & 1.92 & 0.01 & 1.93 & 1.95 & -0.02 \\
\hline 7 & 1.40 & 1.41 & -0.01 & 1.44 & 1.45 & -0.01 \\
\hline 8 & 1.10 & 1.10 & 0.00 & 1.08 & 1.08 & 0.00 \\
\hline 9 & 1.08 & 1.09 & -0.01 & 1.06 & 1.06 & 0.00 \\
\hline 10 & 1.95 & 2.10 & -0.15 & 1.97 & 2.14 & -0.17 \\
\hline \multicolumn{3}{|c|}{ MEAN } & -0.03 & & & -0.02 \\
\hline \multicolumn{3}{|c|}{ SD } & 0.06 & & & 0.06 \\
\hline \multicolumn{3}{|c|}{ p value } & 0.19 & & & 0.29 \\
\hline
\end{tabular}

Table-5: Target minimum dose difference between the smoothening levels of s25 and s75 for PBC and AAA algorithms for the grid size of $\mathbf{0 . 2 5}$.

\begin{tabular}{|c|c|c|c|c|c|c|}
\hline \multirow{2}{*}{ S.No. } & \multicolumn{5}{|c}{ PBC } & \multicolumn{2}{c|}{ AAA } \\
\cline { 2 - 7 } & $\mathbf{s 2 5}(\%)$ & $\mathbf{s 7 5}(\%)$ & Difference & s25 $(\%)$ & s75 (\%) & Difference \\
\hline 1 & 89.20 & 89.30 & -0.10 & 89.00 & 88.40 & 0.60 \\
\hline 2 & 82.10 & 80.50 & 1.60 & 84.30 & 83.60 & 0.70 \\
\hline 3 & 88.70 & 89.80 & -1.10 & 86.30 & 86.70 & -0.40 \\
\hline 4 & 83.80 & 83.30 & 0.50 & 84.20 & 83.70 & 0.50 \\
\hline 5 & 77.60 & 77.70 & -0.10 & 78.60 & 79.60 & -1.00 \\
\hline 6 & 88.20 & 87.80 & 0.40 & 87.60 & 87.80 & -0.20 \\
\hline 7 & 92.90 & 92.30 & 0.60 & 87.60 & 87.30 & 0.30 \\
\hline 8 & 81.70 & 81.50 & 0.20 & 81.30 & 80.50 & 0.80 \\
\hline 9 & 84.50 & 84.80 & -0.30 & 85.40 & 85.50 & -0.10 \\
\hline 10 & 95.50 & 95.70 & -0.20 & 95.40 & 95.70 & -0.30 \\
\hline
\end{tabular}


Original Research Article

Table-6: Target minimum dose difference between the smoothening levels of s25 and s75 for PBC and AAA algorithms for the grid size of 0.5 .

\begin{tabular}{|c|c|c|c|c|c|c|}
\hline \multirow{2}{*}{ S.No. } & \multicolumn{5}{|c}{ PBC } & \multicolumn{2}{c|}{ AAA } \\
\cline { 2 - 7 } & $\mathbf{s 2 5}(\%)$ & $\mathbf{s 7 5}(\%)$ & Difference & s25 $(\%)$ & s75 (\%) & Difference \\
\hline 1 & 88.40 & 88.50 & -0.10 & 83.90 & 84.80 & -0.90 \\
\hline 2 & 82.00 & 80.20 & 1.80 & 75.00 & 73.40 & 1.60 \\
\hline 3 & 85.40 & 84.40 & 1.00 & 84.60 & 86.20 & -1.60 \\
\hline 4 & 84.10 & 83.50 & 0.60 & 83.50 & 84.10 & -0.60 \\
\hline 5 & 78.30 & 77.50 & 0.80 & 75.80 & 76.20 & -0.40 \\
\hline 6 & 87.90 & 88.10 & -0.20 & 87.80 & 86.40 & 1.40 \\
\hline 7 & 90.50 & 90.20 & 0.30 & 87.40 & 87.20 & 0.20 \\
\hline 8 & 81.50 & 81.20 & 0.30 & 76.60 & 77.10 & -0.50 \\
\hline 9 & 83.20 & 83.60 & -0.40 & 84.20 & 84.10 & 0.10 \\
\hline 10 & 95.90 & 95.80 & 0.10 & 95.40 & 95.50 & -0.10 \\
\hline & MEAN & & 0.42 & & & -0.08 \\
\hline
\end{tabular}

Table- 7: Comparison of percentage of pixels passing gamma between the smoothening levels of s25 and s75 for PBC and AAA algorithms for the grid size of 0.25 using I'MatriXX fluence measurement.

\begin{tabular}{|c|c|c|c|c|}
\hline \multirow{2}{*}{ S.No. } & \multicolumn{2}{|c|}{ PBC } & s25 (\%) & s75 (\%) \\
\cline { 2 - 5 } & $\mathbf{s 2 5}(\%)$ & $\mathbf{s 7 5}(\mathbf{\%})$ & 98.26 & 97.13 \\
\hline 1 & 97.09 & 97.58 & 98.49 & 97.37 \\
\hline 3 & 95.70 & 96.22 & 96.82 & 96.56 \\
\hline 4 & 95.69 & 96.47 & 97.55 & 97.14 \\
\hline 5 & 96.80 & 97.53 & 98.24 & 96.83 \\
\hline 6 & 97.37 & 97.43 & 96.64 & 99.02 \\
\hline 7 & 95.93 & 95.75 & 99.75 & 99.11 \\
\hline 8 & 99.41 & 99.58 & 97.89 & 98.65 \\
\hline 9 & 98.20 & 98.62 & 96.40 & 98.02 \\
\hline 10 & 97.78 & 97.85 & 98.07 & 1.09 \\
\hline MEAN & 96.98 & 96.70 & 97.81 & 1.00 \\
\hline SD & 97.10 & 97.37 & & 0.57 \\
\hline value & 1.17 & 1.15 & & \\
\hline
\end{tabular}

The mean difference in HI $(\%)$ were $0.07 \pm 0.31(\mathrm{p}=0.49)$ and $0.07 \pm 0.29(\mathrm{p}=0.47)$ for 0.25 calculation grid size of $\mathrm{PBC}$ and AAA respectively as shown in Table 1 . Similarly for the grid size of 0.5 , the mean differences were $-0.16 \pm 0.55$ $(\mathrm{p}=0.38)$ and $-0.19 \pm 0.50(\mathrm{p}=0.26)$ as in Table 2. The mean difference in CI index were $-0.02 \pm 0.04(\mathrm{p}=0.20)$ and $0.02 \pm 0.03(\mathrm{p}=0.05)$ for the grid size of 0.25 for PBC and AAA respectively as in Table 3 . For the grid size of 0.5 , the changes were $-0.03 \pm 0.06(\mathrm{p}=0.19)$ and $-0.02 \pm 0.06(\mathrm{p}=0.29)$ as shown in Table 4 . The mean difference $(\%)$ of target (PTV) minimum doses were $0.15 \pm 0.71(\mathrm{p}=0.52)$ and $0.09 \pm 0.589(\mathrm{p}=0.64)$ for the grid sizes of 0.25 of PBC and AAA 
algorithms respectively as in Table 5. For the grid size of 0.5 the mean differences were $0.42 \pm 0.66(\mathrm{p}=0.07)$ and $0.08 \pm 0.98(\mathrm{p}=0.80)$ as shown in Table 6 . The quantitative analysis of delivery accuracy from the I'matri XX measurements have been compared in terms of $\gamma$ analysis between s 25 and s75 fluence levels for the grid size of 0.25 for $\mathrm{PBC}$ and AAA algorithms as shown in table 7. The mean percentage of pixel passing set gamma criterion of $3 \mathrm{~mm}$ and $3 \%$ DD were $97.10 \pm 1.17 \mathrm{Vs} 97.37 \pm 1.15(\mathrm{p}=0.05)$ for $\mathrm{PBC}$ and $97.81 \pm 1.00 \mathrm{Vs} 98.02 \pm 1.09(\mathrm{p}=0.57)$ for AAA algorithms.

\section{Discussion}

Several authors suggested as a recommended solution to systematically adopt planning tools and methods able to optimise smooth beam fluences [8-11]; Coselman et al [12] underlined that smoothing algorithms that are applied post-optimisation, usually result in a degradation of the plan according to the objective function while, when the smoothing is part of the objective function, better results are obtainable.

In this study we tried to evaluate the IMRT treatment planning in terms of change in total monitor units, plan quality and delivery accuracy when the plan smoothening parameters are changed. In the Eclipse TPS, both structure-dose-priority weights and smoothing (X, Y) penalty weights are embedded components within the objective function and, thus, contribute to the total score for a particular plan. Therefore, by varying one set of parameters relative to the other, the user can change the ratio of the two penalty components to each other and, therefore, the relative contribution of the smoothing penalty versus the structure-dose penalty. This is important to realize for the Eclipse system because any characterization of the behavior of various smoothing levels must, therefore, be associated with the range of structuredose-priority weights used for that plan.

Anger et al. have evaluated the behavior of three ITPSs when varying smoothing parameters and concluded that, Depending on the inverse treatment planning system used, the potential benefits of optimizing fluence smoothing levels can be significant, allowing for increases in either delivery efficiency or plan conformality [13]. Webb proposed [14], as a general rule of thumb for good IMRT practice, to avoid excessive complexity and, as a metric to appraise the degree of modulation in a fluence matrix, introduced the concept of Modulation Index, (MI) [15].

Excessive modulation leads to high numbers of MUs necessary to deliver prescribed doses with potential consequences on long term effects as secondary cancer induction [16], on treatment time for individual fractions (possibly to relate to organ movement and biological issues) and on radiation protection items. Excessive complexity in the fluence could have a negative trade-off also against inter-fraction tumour dynamics (e.g. hypoxic conditions, tumour stem cells migration, etc) that could be incorporated in the planning strategies [17]. These considerations should be linked to a rather old but still valid note of caution published by Goitein and Niemierko [18] where they proved the principle that the risk of treatment failure is more linked to dose deficits (severe under-dosages to small volumes) rather than to small/ moderate underdosages to larger volumes.

A final concern that could raise from this study is the possibility to determine a "proper" or "necessary" amount of modulation necessary to obtain an high quality plan. This can be hardly achieved by any study since it is obvious that, visible from the data shown here, the relation between smoothening of fluence with their outcomes would helpful for anyone. From the Varian Medical Systems user manual for Eclipse, it was evaluated "no smoothing" $(\mathrm{X}=0, \mathrm{Y}=0)$, "moderate smoothing" ( $X=40, Y=30)$, and "heavy smoothing" $(\mathrm{X}=90, \mathrm{Y}=90)$ for a head and neck plan phantom [19]. Although the actual structure-dose-priority weights used were not specified for this particular analysis, the authors recommended using between 0 and 100 because they historically used this range in an older ITPS (CAD Plan).

This recommendation was not reported to be based on any empirical testing of values outside that range. The plan with "no smoothing" resulted in good target coverage, but they noted "islands of hot spots." From our present study it was noted that, there was a maximum reduction of about $18 \%$ in total monitor units while moving from s25 to s100 level. It was found that there was no statistical difference in $\mathrm{HI}$ index, $\mathrm{CI}$ index, PTV coverages, OAR's like Spinal cord, Bladder, Brainstem \& Rectum doses when the smoothening parameters were changed.

\section{Conclusion}

Commercially available intensity-modulated radiation therapy, inverse treatment planning systems (ITPS) typically include a smoothing function which allows the user to vary the complexity of delivered beam fluence patterns. Fluence complexity is strongly interconnected 


\section{Original Research Article}

to the quality and efficiency of dose delivery (and consequently also to radiation protection issues). There have been limited publications related to variations of fluence levels in IMRT planning and its outcome. Hence, an attempt was made to find the variation between different levels of fluence by the combination of calculation grid size, and different algorithms.

From our present study on the influence of various levels of fluence smoothening in IMRT plan results, it was found that when we increase the smoothening level to higher level, there was a reduction in total MU's with no significant statistical variation in terms of mean differences of HI Index, CI index, PTV coverage, OAR doses. Moreover, in IMRT the reduction in Monitor units will help in reducing head leakage dose hence the lower whole body dose, which will help the patient to reduce the chances for secondary malignancies.

Hence, we conclude that, higher fluence smoothening levels with acceptable difference in target coverage and minimum variation of OAR should be selected for the execution. Based on the our findings, we recommended the workflow for eclipse TPS for IMRT to best exploit the fluence-smoothing features of the system and also this also be a guideline regarding what can be expected when smoothing/efficiency is altered within the Eclipse treatment planning systems.

Funding: Nil, Conflict of interest: None Permission of IRB: Yes

\section{References}

1. Hanušová T, Vondráček V, Badraoui K, Horáková I, Koniarová I. New Method For Estimation of Fluence Complexity in IMRT Fields; Lékař a technika: 2016, 46 (1), 29-32.

2. Bortfeld T, Jokivarsi K, Goitein M, et al. Effects of intra-fraction motion on IMRT dose delivery: statistical analysis and simulation. Phys Med Biol. 2002 Jul 7;47 (13) : 2203-20.

3. Dogan N, King S, Emami B, et al. Assessment of different IMRT boost delivery methods on target coverage and normal-tissue sparing. Int $\mathrm{J}$ Radiat Oncol Biol Phys. 2003 Dec 1;57(5):1480-91.

4. Fogliata A, Bolsi A, Cozzi L. Comparative analysis of intensity modulation inverse planning modules of three commercialtreatment planning systems applied to head and neck tumour model. Radiother Oncol. 2003 Jan; 66 (1):29-40.
5. Kam MK, Chau RM, Suen J, et al. Intensitymodulated radiotherapy in nasopharyngeal carcinoma: dosimetric advantage overconventional plans and feasibility of dose escalation. Send toInt J Radiat Oncol Biol Phys. 2003 May 1;56(1):145-57.

6. Nicolini, G., Fogliata, A., Vanetti, E., Clivio, A., Ammazzalorso, F., Cozzi, L. What is an acceptably smoothed fluence? Dosimetric and delivery considerations for dynamic sliding window IMRT. Radiat. Oncol., 2007, vol. 42, no. 2, p. 42-54.

7. Spirou SV, Fournier-Bidoz N, Yang J, et al. Smoothing intensity-modulated beam profiles to improve the efficiency of delivery. DOI:10.1118/1. 1406522

8. Breedveld S, Storchi PR, Keijzer M, Heijmen BJ. Fast, multiple optimizations of quadratic dose objective functions in IMRT. DOI:10.1088/0031-9155/51/14/019

9. Crooks SM, McAven LF, Robinson DF, Xing L. Minimizing delivery time and monitor units in static IMRT by leaf-sequencing. Phys Med Biol. 2002 Sep 7; 47 (17):3105-16.

10. Langer M, Thai V, Papiez L. Improved leaf sequencing reduces segments or monitor units needed to deliver IMRT using multileaf collimators. DOI:10. 1118/1. 1420392

11. Sun X, Xia P. A new smoothing procedure to reduce delivery segments for static MLC-based IMRT planning. DOI:10.1118/1.1713279

12. Coselmon MM, Moran JM, Radawski JD, Fraass BA: Improving IMRT delivery efficiency using intensity limits during inverse planning. Med Phys 2005, 32:1234-1245.

13. Anker CJ, Wang B, Tobler M, et al. Evaluation of fluence-smoothing feature for three IMRT planning systems. J Appl Clin Med Phys. 2010 Apr 16;11(2): 3035 .

14. Webb S The physical basis of IMRT and inverse planning. DOI:10.1259/bjr/65676879

15. Webb $S$ Use of a quantitative index of beam modulation to characterize dose conformality: illustration by a comparison of full beamlet IMRT, fewsegment IMRT (fsIMRT) and conformal unmodulated radiotherapy. Phys Med Biol. 2003 Jul 21; 48 (14): 2051-62 


\section{Original Research Article}

16. Hall EJ, Wuu CS. Radiation-induced second cancers: the impact of 3D-CRT and IMRT. Int J Radiat Oncol Biol Phys. 2003 May 1;56(1):83-8.

17. Rickhey M, Bogner L: Application of the inverse Monte Carlo treatment planning system IKO for an inhomogeneous dose prescription in the sense of dose painting. Z Med Phys 2006, 16:307-312.
18. Goitein M, Niemierko A. Intensity modulated therapy and inhomogeneous dose to the tumor: a note of caution. Int J Radiat Oncol Biol Phys. 1996 Sep 1;36 (2) : 519-22.

19. Mayo C, Urie M. Eclipse IMRT- A Practical Treatment Planning Guide. Las Vagas, NV: Varian Medical Systems; 2004; 24-27.

\section{How to cite this article?}

B. Rekha Reddy, C. Varatharaj, M. Ravikumar, K. M. Ganesh. Effect of fluence smoothening in intensity modulated radiotherapy planning and delivery. Int J Med Res Rev 2018;6(06):292-300.doi:10.17511/ijmrr. 2018.i06.01. 